\title{
Magie und Apokalyptik im Zentrum lukanischer Geschichtsschreibung - Historizität am Beispiel von Apg 13.6-12
}

\author{
SOHAM AL-SUADI \\ Universität Bern, Theologische Fakultät, Länggassstrasse 51, CH-3012 Bern, \\ Switzerland.Email: soham.alsuadi@theol.unibe.ch
}

\begin{abstract}
Acts 13.6-12 has been viewed as highly significant in the debate about the historicity of Acts, since the beginnings of the Pauline mission among the Gentiles are supposed to be rooted here, and the story illustrates Paul's self-understanding as an apostle. On the other hand, signs of literary creativity of the author with regard to the overall theological concept and the controversy about miracles, magic and apocalyptic traditions are clearly seen in this section that seem to contradict the description of the beginnings of the Pauline mission. This paper explores the apparent contradiction in the debate about the historicity of Luke-Acts in general and Acts 13.6-12 in particular, and shows that magic and apocalypticism can be incorporated within the ancient understanding of historical verification.
\end{abstract}

Keywords: Acts, magic, apocalypticism, mission among the Gentiles, historiography

Der Bericht des Acts-Seminars hat es erneut bestätigt: Die Apostelgeschichte kann im 21. Jh. nicht als historisch verifizierbares Dokument verstanden werden und eignet sich daher auch nicht dazu, Rückschlüsse auf die historischen Hintergründe der christusgläubigen Gemeinschaftsgründungen zu ziehen. ${ }^{1}$ Auch der Versuch, die Grundlage der Apostelgeschichte in einem unabhängigen Reisebericht zu suchen, wird von den Autoren des Acts-Seminar Reports weitgehend abgelehnt. Apg 13.6-12, der Abschnitt, der den Beginn der paulinischen

1 Das sog. Acts-Seminar ist ein Forschungsseminar des „Westar Institute” und hat sich zur Aufgabe gemacht, innerhalb von zehn Jahren den historischen Gehalt der Apostelgeschichte zu untersuchen. „The Seminar on the Acts of the Apostles began deliberations in 2001, with the task of going through the canonical Acts of the Apostles from beginning to end and evaluating it for historical accuracy. The goal was to produce a red-letter edition of Acts, following the publication model the Jesus Seminar used in The Five Gospels and The Acts of Jesus. With such a tool in hand, students of the Bible will be better able to address issues of Christian origins." http://www.westarinstitute.org/projects/the-jesus-seminar/ seminar-on-the-acts-of-the-apostles. 
Mission unter den Heiden beschreibt, wird von vielen Interpreten als Abschnitt angesehen, der auf so einen Reisebericht basieren könnte. Aber auch für Apg 13,6-12 heisst es:

After one removes the features of Lukan composition, there is nothing left to the story except the itinerary itself. The existence of an itinerary source, a source that contained very little, if anything, except a list of places Paul visited in their correct order, is no longer tenable as a working hypothesis. Therefore, we conclude that the itinerary in this text is not based on independent, reliable data but rather is most likely the creation of Luke himself. ${ }^{2}$

Die lukanische Handschrift wird vor allem darin gesehen, dass der Abschnitt eine Zusammenfassung der lukanischen Interpretation der paulinischen Mission repräsentiert. Die göttliche Führung durch den Heiligen Geist, der Beginn in der Synagoge, seine Auseinandersetzungen mit jüdischen Kritikern und die kontinuierliche Konversion von (einflussreichen) Heiden stehen ebenso für die lukanische Kreativität, wie die Wundergeschichte in der Mitte von Apg 13.6-12. Für das Acts Seminar steht also fest: „The entire narrative, therefore, is manifestly a product of Luke's own creativity." ${ }^{3}$ Im Sinne der Mehrheitsmeinung hält Richard Pervo bereits in seinem Kommentar zur Apostelgeschichte fest: "If it is conceded that there is no visible traditional basis for a mission of Paul and Barnabas to Cyprus, it follows that the entire episode is, as it stands, unhistorical." ${ }^{4}$

Folgt man dieser Interpretation, dann werden Anzeichen der lukanischen literarischen Kreativität in dem theologischen Gesamtkonzept und der Thematisierung von Wundern, Magie und Apokalyptik gesehen, da diese den Ansprüchen moderner Historizität nicht genügen. Dennoch wird Lukas, dem Autor des Evangeliums und der Apostelgeschichte, schon allein wegen seines eigenen Anspruchs, das, was geschehen und in Erfüllung gegangen ist aufzuschreiben (Lk 1.1-4), ein grosses historiografisches Interesse unterstellt. So wird auch Apg 13.6-12 im Zusammenhang mit der Historizitätsdebatte ein hoher Stellenwert zugesprochen, da hier die Anfänge der paulinischen Mission unter den Heiden vermutet werden und die Erzählung das paulinische Selbstverständnis illustriert.

Dieser Aufsatz wird dem Widerspruch in der Historizitätsdebatte bezüglich des lukanischen Doppelwerks im Allgemeinen und Apg 13.6-12 im Besonderen nachgehen und zeigen, dass Magie und Apokalyptik durchaus im antiken Sinn von historischer Verifikation verstanden werden können. Im Folgenden werden Lukas und sein historisches Interesse näher untersucht, ehe auf die

2 D. E. Smith und J. B. Tyson, Hg., Acts and Christian Beginnings: The Acts Seminar Report (Salem: Polebridge Press, 2013) 148.

3 Ibid., 148.

4 R. I. Pervo, Acts : A commentary (Hermeneia Minneapolis: Fortress, 2009), 323. 
Informationen und Beschreibungen von Ereignissen in Apg 13.6-12 eingegangen wird. Da Lukas als historischer Erzähler der Anfänge der paulinischen Heidenmission wahrgenommen wird, wird im Anschluss nach Anzeichen für historiografisches Schreiben in Apg 13.6-12 gefragt. Die Frage nach der theologischen Verortung historisch plausibler Informationen in Apg 13.6-12 und detaillierte Studien zu Apokalyptik und Magie in theologischer Geschichtsschreibung leiten in abschliessende Bemerkungen über.

\section{Lukas' historisches Interesse}

Dass das historische Interesse des Autors nicht bedeutet, dass den lukanischen Schilderungen der frühchristlichen Identitäts- und Gemeinschaftsentwicklung hohe historische Authentizität zugesprochen werden kann, ist als Mehrheitsmeinung in den neutestamentlichen Studien vorzufinden. Erkennbar wird das nicht zuletzt an der Beurteilung der vermeintlichen Beziehung zwischen Lukas und dem Apostel Paulus. Lukas wird seit Irenaeus von Lyon unter dem Einfluss des Werdegangs des Apostels Paulus betrachtet. Als Begleiter und Mitarbeiter des Paulus, hätte Lukas, so Irenaeus, das Evangelium schriftlich fixiert (Adv. Haer. 3.1.1; 3.14.1). ${ }^{5}$ Die Wir-Passagen der Apostelgeschichte und das neutestamentliche Bild, das sowohl von Paulus selbst als auch von deuteropaulinischen Schriften von einem Lukas gezeichnet wird (Phlm 24; Col 4.14; 2 Tim 4.11), legen diese Zusammenhänge durchaus nah. ${ }^{6}$ Rudolf von Bendemann bemerkt zu dem Verhältnis zwischen Lukas und Paulus allerdings, dass „L. dabei genauer einem literarischen Motivzusammenhang eingegliedert wird, nach dem Paulus nahezu allein leidet, jedoch bis zur letzten Minute seines Wirkens apostolisch aktiv ist." ${ }^{7}$ Das Bild des Paulusbegleiters Lukas in einen grösseren Motivzusammenhang einzuordnen, in dem vor allem die Missionstätigkeit des Apostels beschrieben wird, verändert die Debatte. Bendemanns Blickwinkel zeigt nicht zuletzt, dass literarische Intentionen frühchristlicher Autoren nicht mit historischer Plausibilität zu verwechseln sind. Die Diskussion über eine umfassende oder partielle Begleitung des Apostels oder gar die Negierung eines möglichen Kontaktes zwischen Paulus und dem Autor des Evangeliums und der Apostelgeschichte wird sich durch die literarischen Anhaltspunkte kaum klären lassen. ${ }^{8}$ Was allerdings allein auf der

5 Vgl.: E. K. Ch. Wong, Evangelien im Dialog mit Paulus: Eine intertextuelle Studie zu den Synoptikern (Göttingen : Vandenhoeck \& Ruprecht, 2012) 40.

6 R. Bendemann, „Lukas”, Das Reallexikon für Antike und Christentum (Hg. E. Dassmann; Stuttgart: Anton Hiersemann, 2010) 647-8.

7 Ibid., 648. Das bedeutet freilich nicht, dass Lukas kein bzw. kein partieller Begleiter des Paulus gewesen sein könnte.

8 Ibid., 648-54 fasst die Argumente kurz zusammen. 
literarischen Ebene deutlich wird, ist klar: Historisch plausible Beziehungen und Entwicklungen in der Gemeinschaftsbildung zu beschreiben ist ein sorgfältig ausgearbeitetes Konzept, das Lukas in seinen beiden Schriften in Bezug auf Personen und ihre Handlungen anwendete.

Wenn es sich um ein sorgfältig ausgearbeitetes Konzept handelt mit dem Lukas Personen und ihre Handlungen beschreibt, dann stellt sich zuerst die Frage nach der Historizität dieser Personen und ihrer Handlungen. Die genannten Personen in Apg 13.6-12 wurden auf ihre Historizität hin überprüft. Wilfried Eckey hält fest, dass

der Statthalter Sergius Paulus wahrscheinlich eine historische Gestalt [ist]. Der Magier Barjesus in der Nähe des Statthalters dürfte ebenfalls historisch sein. Astrologisch arbeitende Wahrsager und Zeichendeuter im Umkreis hochgestellter Persönlichkeiten waren üblich, jüdische Abwehrversuche der Evangelisation verbreitet. Die Wundertradition dürfte zuerst in einer Gemeinde auf Zypern oder in Antiochien erzählt und von Lukas stilistisch gestaltet worden sein. ${ }^{9}$

Trotz dem, dass der Text auf die „üblichen” Verhältnisse eines Statthalters hinweist, reichen die Informationen für andere nicht aus, um Sergius Paulus eindeutig zu identifizieren. Rainer Metzner betont, dass „die Identifizierung des in Apg 13 genannten Sergius Paulus in außerneutestamentlichen Quellen schwierig [ist], da der Vorname (praenomen) des Mannes, der mit Sergius den Familiennamen einer alten patrizischen Familie trägt, nicht genannt ist". ${ }^{10}$ Markus Öhler hält in diesem Zusammenhang fest, dass der inschriftliche Befund eine absolute Datierung nicht zulässt, aber dennoch deutlich wird, dass ein Zusammentreffen der Apostel mit einem Prokonsul Sergius Paulus durchaus möglich war. ${ }^{11}$ Das führt ihn zu dem folgenden Ergebnis:

Einen gewissen Erfolg hatten die Apostel daher m.E. schon, und es ist nicht allein lk. Interesse an römischer Sympathie für das Christentum zuzuschreiben, daß Sergius Paulus in der Erzählung auf der Seite der Christen begegnet. Erreicht hatten sie diesen Erfolg anscheinend mit ihrer Predigt, wenn die Wundertradition tatsächlich sekundär von $\mathrm{Lk}$ in die Geschichte eingebaut

9 W. Eckey, Die Apostelgeschichte: Der Weg des Evangeliums von Jerusalem nach Rom (Neukirchen-Vluyn: Neukirchener, 2000), 282-3.

10 R. Metzner, Die Prominenten im Neuen Testament: Ein prosopographischer Kommentar (Novum Testamentum et orbis antiquus/Studien zur Umwelt des Neuen Testaments; Göttingen 2008), 409.

11 M. Öhler, Barnabas: Die historische Person und ihre Rezeption in der Apostelgeschichte (WUNT; Tübingen: Mohr Siebeck, 2003) 284. Um die Frage nach der Historizität endgültig zu klären schlägt Öhler vor zuerst, nach der Historizität einer Zypernmission zu fragen, um dann die daran beteiligten Personen zu überprüfen und schliesslich nach dem Erfolg dieser Reise zu fragen (Öhler, Barnabas, 285). 
worden ist, und der Hinweis, daß der Prokonsul von der Lehre des Herrn beeindruckt gewesen wäre (v.l2b), zutreffen sollte. Der Beginn der planmäßigen Heidenmission war so wohl kein Riesenerfolg, scheiterte aber nicht vollständig. ${ }^{12}$

An Öhlers Interpretation wird deutlich, dass das Zusammenspiel von historisch wahrscheinlichen und literarisch überzeugenden Anteilen in Apg 13.6-12 dazu führt, dass die Erzählung als „historisch” wahrgenommen werden kann. Öhler macht die Historizität aber nicht unbedingt an den Personen fest, sondern an den Ereignissen. Der Beginn der Heidenmission, der historisch ausser Frage steht, wird von ihm als Verifikation hinzugezogen. Das sorgfältig ausgearbeitete Konzept von Lukas führt dazu, dass sich Historizität nicht an konkreten Personen festmachen lässt, sondern dass eine überzeugenden Kombination aus informierenden und beschreibenden Sachverhalten eine historisch plausible Rekonstruktion der urchristlichen Gemeinschaftsbildung erlauben. Wenn es folglich um die Nacherzählung von Ereignissen geht in denen Personen und ihre Handlungen beschrieben werden, dann weitet sich der Fragehorizont erneut aus. Welche Themen des frühen Christentums, welche literarischen Motive, welche Argumentation vermitteln dem Leser, dass es sich um ein historisch plausibles Ereignis handelt? Am Beispiel von Apg 13.6-12 kann das Zusammenspiel von historisch wahrscheinlichen und literarisch überzeugenden Anteilen dargestellt werden.

\section{Informationen und Beschreibungen von Ereignissen in}

\section{Apg 13.6-12}

Apg 13 beschreibt den Anfang der paulinischen Mission in vielerlei Hinsicht. In seiner ersten Missionsreise wird Paulus, zusammen mit Barnabas und weiteren Begleitern, von Antiochia in Syrien vom Heiligen Geist ausgesandt (13.1-3). Obgleich Barnabas und Paulus nach Apg 13.1 zu einer Gemeinde gehören, in der es Propheten und Lehrer gab, werden sie während eines Gottesdienstes vom Heiligen Geist „für das Werk” bestimmt, zu dem sie vom Geist berufen wurden (Apg 13.2). Dass es sich bei „dem Werk” um die Verkündigung des Wortes Gottes handelt, wird aus Apg 13.5 deutlich: In Salamis auf Cypern (Apg 13.4-5) verkündigen sie in den Synagogen der Juden

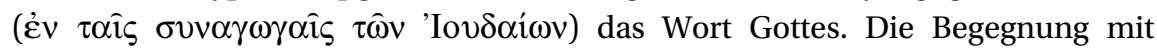
dem jüdischen Magier Barjesus und die Bekehrung des Stadthalters Sergius Paulus finden auch auf Cypern in Paphos statt und werden im Anschluss an die Verkündigung in der Synagoge von Salamis erzählt (Apg 13.6-12). Die Bekehrung des Statthalters Sergius Paulus ist in Apg 13 der erste Hinweis auf die paulinische Mission unter den Heiden. Proklamiert wird die Heidenmission aber erst in Apg 13.46-9. Zuvor reisen Paulus und seine Begleiter von Paphos 
weiter nach Perge in Pamphylien und von dort weiter nach Antiochia in Pisidien (13.14-52). Dort besuchen sie am Sabbat die Synagoge (Apg 13.14), wobei Paulus von dem Vorsteher der Synagoge darum gebeten wird, Worte des Zuspruchs an die Gemeinschaft zu richten. Es folgt in Apg 13.16-43 eine Nacherzählung der „Geschichte Israels”, in der Paulus den Bogen von der Erwählung des Volkes (Apg 13.16-17) über das Hervorgehen Jesu als Nachkomme Davids (Apg 13.23) bis zur Vergebung der Sünden durch Jesus (Apg 13.38-9) spannt. Teil der Nacherzählung ist, dass Johannes als Wegbereiter Jesu beschrieben wird (Apg 13.24-6) und die Bewohner Jerusalems und ihre führenden Männer für den Tod Jesu und damit auch für die Erfüllung der prophetischen Verheissung verantwortlich gemacht werden (Apg 13.27-8). Die Botschaft wird von Paulus am nächsten Sabbat nochmals verkündigt, wobei dort nicht nur die Mitglieder der Synagoge, sondern fast die ganze Stadt versammelt ist (Apg 13.42, 44). Apg 13.45 leitet $\mathrm{zu}$ der Proklamation der Heidenmission über, indem von eifersüchtigen Juden berichtet wird, die Paulus widersprechen (Apg 13.45). Das nimmt Paulus zum Anlass, sich von der Verkündigung des Wortes an die Juden abzuwenden und zu erklären, dass sie sich nun den Heiden zuwenden werden (Apg 13.46-7). Dem Widerspruch und der Verfolgung durch die Juden in Antiochia wird die grosse Freude der Heiden über das Wort Gottes entgegengesetzt (Apg 13.48-9). Hinzu kommt auch, dass im Zusammenhang mit der Weiterreise von Paulus und Barnabas berichtet wird, dass Jüngern von Freude und dem Heiligen Geist erfüllt werden (Apg 13.51-2). Mit dem Bezug auf den Heiligen Geist schliesst sich in Apg 13 der Kreis von der Wirksamkeit desselben. $\mathrm{Zu}$ Beginn ist es der Geist, der Paulus und seine Begleiter auf die erste Missionsreise sendet (Apg 13.1-3) und am Ende des Kapitels werden die Jünger mit Heiligem Geist erfüllt. Während die Opposition der Juden bereits in der kurzen „Geschichte Israels” von Paulus als notwendige Voraussetzung für die Erfüllung der Verheissung angekündigt und darauf folgend auch von ihm und seinen Begleitern erlebt wird, ist die Bekehrung des Statthalters Sergius Paulus nicht mit der folgenden Mission unter den Heiden verbunden. Ungeachtet dessen, dass die erste Bekehrung eines Heiden keine Kaskade weiterer Bekehrungen auslöst, fungiert Apg 13.12 als Schlüsselstelle für das Verstehen der paulinischen Mission.

In Apg 13.6-12 kommen viele Handlungsstränge zusammen: Barnabas und Paulus treten auf der ersten Missionsreise als Exorzisten und Heiler in Erscheinung, was sich durchaus mit den Informationen aus 2 Kor 12.12; Röm 15.19 und Lk 10.9 par. vergleichen lässt. ${ }^{13}$ Stefan Schreiber beschreibt den Handlungsverlauf der Geschichte als stringent. ${ }^{14}$

13 R. W. Gehring, Hausgemeinde und Mission: Die Bedeutung antiker Häuser und Hausgemeinschaften von Jesus bis Paulus (BWM 9; Gießen: Brunnen, 2000) 204.

14 Schreiber, Wundertäter, 24. 
Paulus und Barnabas treffen einen jüdischen Magos und Lügenpropheten Barjesus/Elymas am Hofe des römischen Statthalters Sergius Paulus, wobei letzterer von ihnen das Wort Gottes hören will. Der Magos leistet ihnen Widerstand, Paulus schilt ihn und kündigt ihm die Strafe der Blendung an, die sofort eintritt. Daraufhin wird der Statthalter gläubig. Eine gewisse Schwierigkeit bietet die abrupte Einführung des Namens Elymas für den vorher Barjesus Genannten, ebenso der Namenswechsel Saulus-Paulus. Es erscheint eine Reihe von Begriffen, die sich als Gegensätze lesen lassen: Barjesus - Sohn des Teufels, voll heiligen Geistes - voll aller List und Gaunerei, verständig - Feind aller Gerechtigkeit. Auffällig ist ferner, daß Barnabas, der noch in V. 7 mit Paulus genannt ist, in der weiteren Handlung keine Rolle mehr spielt. ${ }^{15}$

Das übergeordnete Interesse des Abschnittes ist es aber, den Beginn der Mission unter den Heiden zu beschrieben. Dennis Smith und Joseph Tyson halten im Bericht des Acts-Seminars fest, dass in Apg 13.6-12 das paulinische Vorhaben, erst unter den Juden und dann unter den Heiden das Evangelium zu verkünden (Röm 1.16), umgesetzt wird. ${ }^{16}$ Zwar spart der Abschnitt die Reaktion der Juden in der Synagoge aus, aber Barnabas und Saul reisen von Salamis nach Paphos, wo sie den Prokonsuls Sergius Paulus treffen, der sich zum Christusglauben bekennt. ${ }^{17}$ Die verwirrende Namensgebung der Protagonisten, wird als wichtiger Hinweis auf das historiographische Interesse des Autors verstanden: (1) Saulus wird nach Apg 13.9 nur noch Paulus genannt, wenn es sich nicht um Rückblicke auf seine Konversion handelt; (2) Der Magier Bar-Jesus wird auch als Elymas vorgestellt, was einer griechischen Übersetzung entsprechen soll (Apg 13.8); (3) der Prokonsul wird als Sergius Paulus vorgestellt, was Paulus stärker in der Heidenmission verortet. ${ }^{18}$ Die umfassende Nennung von griechischen und römischen Namen ist an sich nicht aussergewöhnlich - in Apg 13.6-12 sticht sie allerdings heraus, da sie zusammen mit der Mission unter den Heiden genannt wird und sich damit auch ein Wechsel in den Missionsfeldern verbindet. In Apg 13.6-12 werden mit den Namenangaben also

15 Ibid., 24.

16 Smith und Tyson, Acts, 146.

17 Ibid., 146.

18 Ibid., 147-8. Maia Kotrosits macht in ihrem Beitrag zur christlichen Identitätsentwicklung auch darauf aufmerksam, dass zwischen Paulus und dem römischen Prokonsul trotz der Annäherung durch die Namensgebung politische Hierarchien abgebildet werden. Sie zitiert K. Yamazaki-Ransom, The Roman Empire in Luke's Narrative (London: T \& T Clark, 2010) 119: „Luke's use of the double name does not merely signify the Greco-Roman setting of the narrative, it also indicates the socio-political relationship between Sergius Paulus and Paul. The use of two names for an individual, one in his native language, the other in the language of the colonizers, points to the reality of the imperial domination of other cultures. In Luke's narrative, this is Paul's first encounter with a Roman official, and therefore the use of his Roman name is appropriate ... Thus the relationship of Sergius Paulus and Paul is that of colonizer and colonized." 
vor allem Wendepunkte beschrieben: (1) Saulus wird mit der Heidenmission zum Paulus und (2) der Römer Sergius Paulus wird vom Heiden zum Christusgläubigen. Sind sie Wendepunkte in der Erzählung, die über die Namensgebung impliziert werden in diesem Sinn Ausdruck der hellenistischen Geschichtsschreibung des Autors?

\section{Lukas als historischer Erzähler der Anfänge der Heidenmission}

Schon bei der Einordnung des Magiers in der Gefolgschaft von Sergius Paulus wurde die Bedeutung der hellenistischen Kultur im Zusammenhang mit der Rekonstruktion der urchristlichen Gemeinschaftsbildung erörtert. Es steht ausser Frage, dass sich hinsichtlich der sozio-historischen Verortung und der Datierung der beiden Werke der Konsens herausgestellt hat, dass das Evangelium und die Apostelgeschichte vom selben Autoren geschrieben wurde. ${ }^{19}$ Doch die politischen, sozialen und kultischen Einflüsse in den einzelnen Erzählungen lassen sich nicht so einfach eingrenzen. In diesem Sinn verweist Reinhard von Bendemann auf eine „doppelte traditionsgeschichtliche Bildungstiefe" und möchte damit verdeutlichen, dass Lukas sowohl die jüdische als auch die hellenistische Kultur für seine Narrationen berücksichtigt hat. ${ }^{20}$ Obwohl die Einflüsse der römischen Welt von ihm nicht spezifiziert werden, geht Bendemann davon aus, dass solche Unterscheidungen religionshistorische Kategorien sind, die nicht den emischen Verhältnissen des Autors entsprechen. Da die Schrift sich dieser Kategorisierung widersetzt, bleibt dem Interpreten nur, die Hinweise sorgfältig zu kontextualisieren. Um Hinweise bezüglich der sozio-kulturellen Verortung des lukanischen Doppelwerks und seines Autoren zu erlangen, wird vermehrt auf das Genre der Schriften Bezug genommen. Was das Genre und die traditionsgeschichtlichen Einflüsse angeht, versucht Daryl D. Schmidt mit Philip Satterthwaite einen status quo zu etablieren. Satterthwaite fasst zusammen, was $\mathrm{zu}$ den Einflüssen und Traditionen bekannt ist:

19 Eine Entscheidung über den Ort der Entstehung des lukanischen Doppelwerks zu treffen ist zuweilen der Anfang dieser Debatte. Geht man, wie beim Namen des Autoren mit der altkirchlichen Tradition, die die Nähe zwischen der Biographie des Apostels und Lukas in den Vordergrund rückt, dann ist Rom der gewählte Ort der Entstehung (R. Bendemann, 'Lukas', 657). Die Abfassungszeit beider Werke ist ebenso umstritten. Eine Frühdatierung, die begründet, warum der Tod des Apostels nicht erwähnt wird, oder eine Abfassungszeit, die noch keine umfassende Paulusbriefsammlung kennt, lassen sich auf der Basis der ZweiQuellen-Theorie und der vermittelten Zerstörung Jerusalems im Evangelium ausschliessen. Bendemann schliesst auch eine Datierung in die Regierungszeit Domitians aus, da sich die Texte seiner Meinung nach, nicht sicher einem bestimmten politischen Verhältnis zuordnen lassen. Stattdessen sieht er das Evangelium und die Apostelgeschichte nacheinander in der sog. dritten frühchristlichen Generation verfasst (ibid., 658).

20 Ibid., 655-6. 
At point after point Acts can be shown to operate according to conventions similar to those outlined in classical rhetorical treatises. There are some aspects which it is hard to explain other than by concluding that Luke was aware of rhetorical conventions .... The preface, in particular, seems to make an implicit claim to be operating within classical conventions. That is not to say that other influences have not been at work. The Old Testament has obviously exerted considerable influence on Acts; and this may have extended to narrative techniques .... In general it seems fair to speak of a considerable indebtedness to classical rhetoric; that is, he gives clear indication of having received the kind of (rhetorical) education one would expect in a GrecoRoman writer of this period who embarked on a work of this sort. Luke, however, is not a slave of classical conventions .... [The evidence] suggests Luke's freedom in regard to the conventions in which he had been educated, which implies a considerable mastery of those conventions. ${ }^{21}$

Ohne besondere Anhaltspunkte macht Satterthwaite in dieser Zusammenfassung Aussagen über das literarische Genre und überträgt seine Ansichten auf den Autoren. Lukas ist demnach ein Freidenker, der Herr über seine eigene Tradition ist. Auch wenn die Wechselwirkung zwischen Autor und Genre bei Satterthwaite überzeichnet ist, unterstreicht Schmidt, dass Lukas im Spektrum der hellenistischen Geschichtsschreibung zu verorten ist und beschreibt ihn als einen historischen Erzähler und nicht als Historiker. ${ }^{22}$ Verbindet man die Differenzierung zwischen einem historischen Erzähler und einem Historiker mit der Erkenntnis, dass Lukas ein sorgfältig ausgearbeitetes Konzept mit einer überzeugenden Kombination aus informierenden und beschreibenden Sachverhalten gestaltet hat, das eine historisch plausible Rekonstruktion der urchristlichen Gemeinschaftsbildung erlaubt, dann ist folgerichtig anzunehmen, dass Lukas als historischer Erzähler einen Reisebericht des Apostels für seine eigene Interpretation der paulinischen Mission herangezogen hat. Von Interesse ist folglich nicht, ob Paulus Saulus hiess oder ob es einen Prokonsul mit Namen Sergius Paulus gab, der von ihm missioniert wurde. ${ }^{23}$ Im Zentrum steht das Konzept, das historisch plausibel ist. Christoph Müller begründet das „historische Element” der Erzählung nicht mit einer geschulten Rhetorik oder dem geschickten Erzählen sondern damit, dass Lukas „in seinem Werk keinen direkten, sondern einen primär theologisch vorstrukturierten Zugang zur Vergangenheit" vermittelt. ${ }^{24}$

21 D. Schmidt, „Rhetorical Influences and Genre”, Jesus and the Heritage of Israel: Luke's Narrative Claim upon Israel's Legacy (Hg. D. P. Moessner; Harrisburg, PA: Trinity Press International, 1999) 60 zitiert Ph. E. Satterthwaite, „Acts against the Background of Classical Rhetoric", B. W. Winter und A. D. Clarke, The Book of Acts in its Ancient Literary Setting (Grand Rapids: Eerdmans, 1993), 337-9, hier 378-9.

22 Schmidt, „Rhetorical Influences”, 59.

23 Smith und Tyson, Acts, 148-9.

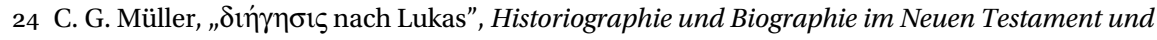
seiner Umwelt (Hg. T. Schmeller; Göttingen: Vandenhoeck \& Ruprecht, 2009) 96. 


\section{Theologische Verortung historisch plausibler Informationen}

Bisher konnte festgehalten werden, dass Lukas historisch plausible Beziehungen und Entwicklungen in der Gemeinschaftsbildung beschreibt, um Personen und ihren Handlungen in der frühchristlichen Gemeinschaftsgründung zu verorten. Die Kombination aus informierenden und beschreibenden Sachverhalten, stellt nicht nur die Frage nach der historischen Einbettung der Ereignisse, sondern auch nach der theologischen Verortung innerhalb eines historisch plausiblen Berichts. Ausschlaggebend ist, dass die lukanische Theologie im Forschungsdiskurs mehrheitlich der jüdischen Tradition zugeordnet wird. Anschaulich wird dies mit dem Begriff der "Geschichtstheologie”, der in Bezug auf Lukas vor allem aufgrund der Monografie von Sylvia Hagene Zeiten der Wiederherstellung: Studien zur lukanischen Geschichtstheologie als Soteriologie an Popularität gewann. Für Hagene steht nicht die antike Historiographie im Vordergrund, sondern die lukanische Soteriologie, die sich ihrer Meinung nach nicht aus der Gegenüberstellung von Sünde und Vergebung entfaltet, sondern auf der Entwicklung von Wissen zur Vergebung basiert. ${ }^{25}$ Nichtsdestotrotz gründet ihr theologisches Konzept auf der Annahme, dass der Autor des Evangeliums und der Apostelgeschichte auf der Basis der apokalyptischen Tradition eine eigenständige Form der Geschichtsschreibung entwickelte. ${ }^{26}$ Ganz im Sinn einer deuteronomistischen Geschichtsschreibung legt ihr Ansatz nahe, dass Lukas die Geschichte der Gottesbegegnung zu seinem Gegenstand gemacht hat. ${ }^{27}$ In diesem Sinn versteht sie Lukas als „Theologen der Heilsgeschichte”. ${ }^{28}$ Auch wenn Lukas bereits in seinem Proömium eine Kontinuität der Heilsgeschichte vermittelt, und damit an der Glaubensgeschichte Israels besonderes Interesse zeigt, muss zu Hagenes Ansatz hinzugefügt werden, dass Lukas, wie andere historiographische Autoren

25 S. Hagene, Zeiten der Wiederherstellung: Studien zur lukanischen Geschichtstheologie als Soteriologie (Neutestamentliche Abhandlungen. Neue Folge 42; Münster: Aschendorff, 2003) 65-6. Hagene bezieht sich auf Aleida Assmanns Studie Zeit und Tradition: Kulturelle Strategien der Dauer (Beiträge zur Geschichtskultur; Köln: Weimar, 1999).

26 Hagene, Zeiten der Wiederherstellung, 53-8. Ibid., 57: „Das von Lukas aus seiner Kenntnis des Markusevangeliums sowie der griechisch-römischen und der jüdischen Geschichtsschreibung zu einer eigenständigen Form entwickelte Geschichtsbild läßt sich letztlich nur vor dem Hintergrund einer apokalyptischen Hermeneutik verstehen."

27 H. Braun, Geschichte des Gottesvolkes und christliche Identität: Eine kanonisch-intertextuelle Auslegung der Stephanusepisode Apg 6,1-8,3 (WUNT 2 279; Tübingen: Mohr Siebeck, 2010) 46.

28 Ibid., 46. Vgl. schon: U. Wilckens, Die Missionsreden der Apostelgeschichte: Form- und traditionsgeschichtliche Untersuchungen (WMANT; Neukirchen-Vluyn: Neukirchener Verlag des Erziehungsvereins, 1963) 204: „Diese Theorie der Heilsgeschichte besteht darin, daß einerseits alle Geschichte in ihrem Wesen als von Gott vorhergeplanter und gewirkter, einlinig-zielgerichteter Geschehenszusammenhang von Verheissung und Erfüllung und dieser zugleich andererseits als im Heilsplan Gottes vorherbestimmter Heilsbereich verstanden wird." 
der Antike, von einem „göttlichen Muss” in der Geschichte ausging. ${ }^{29}$ Mit anderen Worten bedeutet die Verknüpfung von Theologie und Geschichte bei Lukas nicht unbedingt eine kontinuierliche Fortschreibung der deuteronomistischen Geschichtstheologie, sondern eine gezielte Verortung des Wortes Gottes in einer scheinbar vom römischen Reich dominierten Welt. ${ }^{30}$

Da die gezielte Verortung des Wortes Gottes für die Interpretation der lukanischen Theologie von zentraler Bedeutung ist, ist es entscheidend, entsprechende Definitionen von den Begriffen „Theologie” und "Geschichte” bei Lukas zu etablieren. Knut Backhaus und Gerd Häfner leisten in ihrem Aufsatzband Historiographie und fiktionales Erzählen: Zur Konstruktivität in Geschichtstheorie und Exegese einen wichtigen Beitrag, um dem antiken Geschichtsverständnis und der Geschichtsschreibung näherzukommen. Die Autoren erläutern in jeweils zwei Essays die Bedeutung der modernen Geschichtswissenschaft für die neutestamentliche Exegese, wobei der erste Essay die theoretische Diskussion veranschaulicht und der zweite Essay eine Anwendung auf das Neue Testament, namentlich die Apostelgeschichte und die Rückfrage nach Jesus, darstellt. Knut Backhaus' Essay Spielräume der Wahrheit: Zur Konstruktion in der hellenistisch-reichsrömischen Geschichtsschreibung fragt, „inwiefern im antiken, schwerpunktmäßig im späthellenistisch-frühreichsrömischen Diskurs das konstruktive Moment in biographisch-historiographischer Dokumentation reflektiert [wird]". ${ }^{31}$ Entscheidend ist diese Frage deshalb, weil Backhaus davon ausgeht, dass die antike Geschichtsschreibung mit einem Blick in den Spiegel zu vergleichen ist, da sich der Historiograph „eigentlich” selbst sieht, obwohl er ein Fenster bräuchte. Für Backhaus steht daher fest, „dass hellenistisch-reichsrömische Geschichtsschreibung im Hauptstrom ein Mischtypus [ist], der die Rekonstruktion extratextualer Sachverhalte mit ordnenden Konstruktionselementen aus Rhetorik, mimetischer Kunst (Epos, Drama, Roman) und paideutischem Traktat zur narrativen Kohärenz verbindet". ${ }^{32}$

Im Unterschied zum modernen Anspruch dient Geschichtsschreibung in der Antike somit nicht der Dokumentation, sondern der Pflege der funktionalen Gedächtniskultur der Gemeinschaft in ihrer gegenwärtigen Befindlichkeit. ${ }^{33}$ Es handelt sich um ein fiktionales Erzählen, das „zum Selbstverständnis des Wirklichkeitsbegriffes [gehört], da der dokumentierte Sachverhalt gar nicht anders fassbar ist, als in einem konsistenten Sinnsystem, das sich dem steten

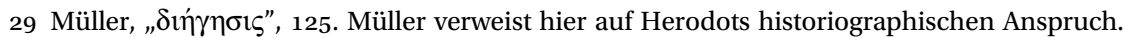

30 Ibid., 123.

31 K. Backhaus und G. Häfner, Historiographie und fiktionales Erzählen: Zur Konstruktivität in Geschichtstheorie und Exegese (Biblisch-theologische Studien; Neukirchen-Vluyn: Neukirchener, 2007) 2.

32 Ibid., 4.

33 Ibid., 27. 
Crossover zwischen Rekonstruktion und Konstruktion verdankt und so einen gewissen Wahrheitsspielraum nachgerade verlangt". ${ }^{34}$

Wenn es also die Intention des Historikers ist, die Erwartungshaltung des Publikums nach einem sinnstiftenden Konzept für die Gegenwart zu erfüllen, dann steckt in dieser Forderung nach Wahrheit nunmehr auch ein konstruktives Element für die Gemeinschaft. ${ }^{35}$ Was sich auf den ersten Blick als ausgeglichene Interpretation der antiken Verhältnisse liest, bekommt bei Backhaus dann doch noch eine differenzierende Wende. Backhaus unterscheidet zwischen paganer Geschichtsschreibung und lukanischer Geschichtsschreibung indem er den paganen Historiographen den Wunsch nach einer unparteilichen und ausgewogenen Darstellung zuschreibt, während Lukas affirmativ und distanzlos sein Geschichtsbild durchsetzen würde. ${ }^{36}$ Hier erweckt Backhaus den Eindruck, dass die Trias aus Erinnerung, Kontinuität und Identität ${ }^{37}$ - die Hagene als Kennzeichen des historischen Erzählens entwickelt - auch bei ihm grundlegend ist und mit einer frühchristlichen Identitätsentwicklung solche Formen der Affirmation und Distanzlosigkeit Rechtfertigung erhalten. Hagene legt in ihren hermeneutischen Grundlagen fest, dass „historische Sachverhalte an sich noch nicht Geschichte sind, sondern erst am Ende eines Prozesses der Sinnbildungsarbeit Geschichte konstruieren".$^{38}$ Historisches Erzählen qualifiziert sich dadurch, dass im Rahmen von Erinnerungen eine Einheit von Vergangenheit, Gegenwart und Zukunft konstruiert wird. In Abgrenzung zum „allgemeinen” Erzählen zeichnet sich das „historische” Erzählen darin aus, dass die Faktoren Erinnerung, Kontinuität und Identität, den inneren Zusammenhang der „Zeitverlaufsvorstellungen” und die „Selbstvergewisserung der historisch Erzählenden" gewährleisten. ${ }^{39}$ Dem Historiker wird mit anderen Worten eine Intentionalität in Hinblick auf sich und seine Leser zugeschrieben, die eine kulturelle Leistung widerspiegelt. Dass es sich bei Lukas um eine „intentionale Geschichtsschreibung" handelt, lässt sich durchaus bestätigen. Ob daraus folgt, dass pagane Autoren weniger intentional bzw. distanziert historische Zusammenhänge beschreiben, muss an einem anderen Ort diskutiert werden. Dort, wo Backhaus auf die Dichte der Erzählung und die sich überschneidenden Sinnzusammenhänge hinweist, ist ihm gerade in Hinblick auf das lukanische Doppelwerk nicht zu widersprechen.

Für die Interpretation von Apg 13.6-12 sind diese Hinweise von entscheidender Bedeutung. Lukas bezieht sich auf ein Ereignis im Verlauf der frühchristlichen Mission, das auf Grund des implizierten Wechsels der religiösen Praxis von

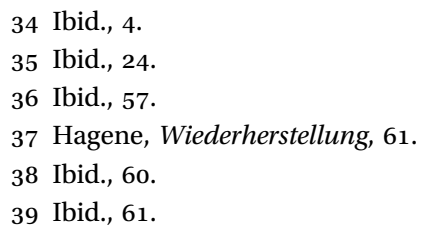


entscheidender Wichtigkeit ist. Die Gedächtniskultur ist in diesem Sinn von einer gemeinschaftsbildenden Funktionalität geprägt, die sich auf die Wende in der Mission bezieht: Saulus wird mit der Heidenmission zum Paulus und der Römer Sergius Paulus wird vom Heiden zum Christusgläubigen.

Da die Anfänge der Mission mit der Auseinandersetzung mit dem Magier Elymas beginnen, der nach der Drohrede des Apostels zeitweise erblindet, liegt es nahe, das übergeordnetes Bild der Trias aus Erinnerung, Kontinuität und Identität in der Thematisierung von Apokalyptik und Magie zu verorten. Erinnerung, weil das Bild des Erblindens bereits aus der Berufung des Apostels bekannt ist - Kontinuität, weil mit der Mission unter den Völkern das Wort Gottes umfänglich verbreitet wird und Identität, weil damit potentiell weitere christusgläubige Gemeinschaften gegründet werden können.

\section{Apokalyptik und Magie in theologischer Geschichtsschreibung}

Apokalyptik und Magie werden in der neutestamentlichen Wissenschaft unter verschiedenen Aspekten diskutiert. Obgleich das Neue Testament nur eine Apokalypse beinhaltet, finden sich zahlreiche Texte, die durch ihren Bezug auf Prophetie, eschatologische Erwartungen oder die Offenbarung von himmlischen Wahrheiten als „apokalyptische Texte” bezeichnet werden können. Christopher Rowland und Judith L. Kovacs halten fest, dass zwischen dem apokalyptischen Genre und der Thematisierung apokalyptischer Motive unterschieden werden muss. ${ }^{40}$ In dieser Hinsicht wurden Mk 13 und 1 Thess 4.16 in der Auslegungsgeschichte als apokalyptische Texte verstanden. Hinsichtlich des lukanischen Doppelwerks werden von Rowland und Kovacs Apg 2; 7.55-6; 10.11; 11.5 und die Berufungserzählungen des Apostels Paulus (Apg 9; 22; 26) zu den apokalyptischen Texten gezählt. In Lk 2.13, 32; 10.21 und 12.2 sehen sie Hinweise auf apokalyptische Terminologie. ${ }^{41}$ Magie wird im Neuen Testament sehr unterschiedlich thematisiert. Zum einen lässt sich eine deutliche Distanz zu Zauberern und Divination feststellen (vgl.: Apg 8.9-24; 13.6-11; 19.19 und 16.16-18) und zum anderen weisen die Heilungen Jesu eine Nähe $\mathrm{zu}$ magischen Praktiken auf (vgl.: Mk 7.31-7; 8.22-6; Joh 9.11-41). ${ }^{42}$ Obschon Jesus nicht dem sozio-kulturellen Bild eines Magiers entspricht, wird er als besessen wahrgenommen (Mk 3.20) und freie Exorzisten instrumentalisieren seinen Namen für Heilungen (Mk 9.38-9; Apg 19.13). Dass die Loslösung von fremder Magie durch Jesus Christus

40 Chr. Rowland und J. L. Kovacs, Apocalypses and Apocalypticism: New Testament (Encyclopedia of the Bible and its Reception (EBR); Berlin, 2009) 341-2.

41 Ibid., 341-2.

42 M. Frenschkowski, Magie: Neues Testament (Religion in Geschichte und Gegenwart; Tübingen, 2002) 668-9. 
ein theologischer Leitgedanke ist, wird in Lk 10.9, 17, 19, Mk 16.17-18 diskutiert. ${ }^{43}$

Bisher wird in der neutestamentlichen Exegese von Apg 13.6-12 wenig beachtetet, dass zu dem historischen Eindruck des Abschnitts die zürnende apokalyptische Rede des Apostels ebenso gehört, wie die Charakterisierung des Magiers als Pseudopropheten. Diese beiden Inhalte führen nicht selten zu Schwierigkeiten in der Interpretation, da Apokalyptik und Magie als transzendierende Themen wahrgenommen werden, die sich dem historiographischen Interesse des Autors widersetzen. Im lukanischen Doppelwerk wird besonders Apg 5.9 und Apg 13 im Zusammenhang mit Apokalyptik und Magie wahrgenommen. So sieht sich Ulrich B. Müller dazu veranlasst die Gerichtsankündigungen in Apg 5.9 und 13.10-11a als Hinweis darauf zu verstehen, dass hier gerade kein historisch plausibles Bild des frühen Christentums vermittelt wird, da Petrus und Paulus in den beiden Szenen nicht als Verkünder, sondern als Richter agieren:

An der Form des jeweiligen Fluchwortes ist zu erkennen, dass an beiden Stellen der Einfluss alttestamentlich-jüdischer Prophetengerichtsreden anzunehmen ist. In Apg 5,9 und 13,10-11a folgt auf die Anklage (Scheltwort) die durch diese begründete Gerichtsankündigung (Drohwort). Beide Male wird die Gerichtsankündigung typisch mit „Siehe” eingeleitet. Von diesen Beobachtungen her könnte man beide Stellen als charakteristische Zeugnisse urchristlicher Prophetie ansehen, die in alttestamentlich-jüdischer Formtradition steht. Nur ist hier große Vorsicht geboten. Rein formal liegt hier der Stil prophetischer Gerichtsrede vor, faktisch aber haben beide Worte innerhalb der jeweiligen Szene die Funktion von Fluchworten, die aufgrund ihrer magischen Kraft das Unheil unmittelbar herbeirufen, also mehr als Ankündigungen sind. Petrus in Apg 5 und Paulus in Apg 13 agieren wie Paulus in 1 Kor 5 nicht mehr nur als Verkünder des Willens Gottes bzw. Christi, sondern schon als Richter selbst, wenngleich der Gerichtsspruch die Form der bloßen Ankündigung bewahrt. ${ }^{44}$

An Müllers Interpretation von Apg 5.9 und 13.10-11a zeigt sich, dass die Rolle von Petrus und Paulus als Richter nicht zu seinem Bild frühchristlicher Prophetie passen, da sie mehr als Ankündigungen sind. Da beide Fluchworte nicht zu seiner Vorstellung eines frühchristlichen Propheten passen, passen auch Magie und Apokalyptik nicht in das Bild eines historisch plausiblen Erzählens. Was das Bild des Apostels als Richter in Apg 13.10-11a über das lukanische

43 Ibid., 669.

44 U. B. Müller, Prophetie und Predigt im Neuen Testament: Formgeschichtliche Untersuchungen zur urchristlichen Prophetie (Studien zum Neuen Testament 10; Gütersloh: Mohn, 1975) 183. Schreiber, Wundertäter, 27-8 sieht deutliche Differenzen zu sog. Fluchworten. Diese bestehen darin, dass es sich (1) in Apg 13.11 nicht um eine magisch wirkende Macht, sondern um Gott handelt, der sich nicht als doppelpolig erweist, sondern vielmehr im Sinne seiner Botschaft "gut” handelt und (2) das Wort des Paulus nicht als die Strafe wirkt, sondern die „Hand des Herrn". Schreiber bevorzuge daher die Bezeichnung Unheilsansage. 
Verständnis historiographischer Berichtserstattung aussagt, wird von Müller nicht diskutiert. David Aune geht den Ursprüngen der literarischen Komposition nach und legt Wert auf die nähere Beschreibung des Fluchwortes:

In Acts 13:9-11 the act of revelation and the transmission of the revelatory message occur at the same instant. This emphasis on the articulation of oracular speech while in a state of inspiration, though rare in the OT (cf. Balaam in Num. 23-24), is more frequent in early Judaism (probably through Hellenistic influence) and pervades Greco-Roman paganism and early Christianity. These considerations suggest that Luke has not modeled Paul's prophetic speech in Acts 13:9-11 directly from OT speech forms. ${ }^{45}$

Aune kommt im Rahmen seiner Frage nach den literarischen Ursprüngen zu der Beobachtung, dass in Apg 13.9-11 der Akt der Offenbarung und die Übertragung der Offenbarung im gleichen Augenblick auftreten. Das ist eine aufschlussreiche Bemerkung, denn sie legt nahe, dass das apokalyptische Fluchwort einen Offenbarungscharakter beinhaltet, der eine bewusste lukanische Konstruktion im Rahmen seines historiographischen Interesses widerspiegelt. Erinnerung, Kontinuität und Identität verbinden sich folglich nicht nur mit der Konversion des Prokonsuls oder dem Beginn der Heidenmission, sondern liegen bereits in der apokalyptischen Rede des Apostels an den Magier. Hinsichtlich Apokalyptik und Magie im Neuen Testament soll nun danach gefragt werden, welche Bedeutungen Apokalyptik und Magie in Apg 13.6-12 beigemessen werden können.

\subsection{Apokalyptik}

Apokalyptik, so stellt sich spätestens nach der Lektüre von Michael Beckers und Markus Öhlers Studien heraus, ist nicht nur in der Moderne eine Herausforderung für das sich bildende und erneuernde Christentum. ${ }^{46}$ Bereits im

45 D. E. Aune, Prophecy in Early Christianity and the Ancient Mediterranean World (Grand Rapids: Eerdmans, 1983) 270.

46 M. Becker und M. Öhler, Apokalyptik als Herausforderung neutestamentlicher Theologie (WUNT 2; Tübingen: Mohr Siebeck, 2006). Jörg Frey fasst treffend zusammen: „Letztendlich dominierte die theologische Skepsis gegenüber der eschatologischen Zukunftserwartung, verbunden mit dem Eindruck, dass die apokalyptischen Texte allzu sehr in eigenmächtigen Bildern und Vorstellungen schwelgten und auch aus diesem Grund weniger auf göttlicher Offenbarung als auf menschlich-allzumenschliche Wunschvorstellungen zurückzuführen seien. Dementsprechend hat sich auch seit der Einführung des fachsprachlichen Terminus ,Apokalyptik' in die theologische Diskussion durch Friedrich Lücke die neutestamentliche Exegese sich oft bemüht, Jesus und die Apostel von dieser jüdischen Strömung und ihrer Geisteshaltung möglichst abzurücken, eben weil man in den apokalyptischen Bildern und noch mehr in den eschatologischen Berechnungen kaum religiös Gültiges erkennen konnte.” J. Frey, „Was erwartet die Johannesapokalypse?”, Die Johannesapokalypse: Kontexte - Konzepte - Rezeption (Hg. J. Frey; Tübingen: Mohr Siebeck, 2012) 476-7. 
neutestamentlichen Kontext ist davon auszugehen, dass die Terminologie

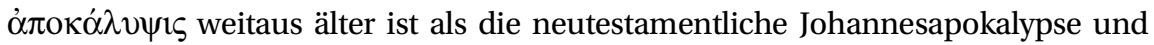
zudem überhaupt nicht einheitlich gebraucht wurde. ${ }^{47}$ Die Vieldeutigkeit ergibt sich daraus, dass das als „Apokalyptik” markierte Problem einerseits spezifischer ist als der allgemeine Vorgang einer Gottesoffenbarung und andererseits breiter ist, als der literarische Niederschlag in den „Apokalypsen”. ${ }^{48}$ Becker und Öhler begründen diesen Sachverhalt damit, dass die apokalyptischen Texte mehr sind, als eine „Selbstkundgabe Gottes oder die Mitteilung bestimmter Offenbarungsworte” ${ }^{49}$ Sie sind „Modelle und Konstruktionen, welche eine Wirklichkeitserfahrung im jeweiligen Offenbarungsgeschehen beschreibbar machen wollen" ${ }^{50}$ Es stellt sich folglich die hermeneutische Frage, wie man die Eschatologie der Apokalypse und damit auch verwandter Texte hermeneutisch beurteilen soll, wenn man diese Elemente neutestamentlicher Schriften für die Exegese heranziehen möchte. ${ }^{51}$

Jörg Frey skizziert vier grundsätzlich voneinander $\mathrm{zu}$ unterscheidende hermeneutische Modelle: die überzeitliche, oder symbolische Interpretation der Apokalypse, die welt- und kirchengeschichtliche Deutung, die von der Abbildung einer geschichtlichen Ereignisfolge ausgeht, die endgeschichtliche Deutung, die einen abgeschlossenen Handlungsverlauf in der Zukunft beschreibt und die zeitgeschichtliche Verortung, die die Apokalypse als Reflexion der Sachverhalte zur Zeit des Autors verstehen will. ${ }^{52}$ Geht man davon aus, dass in der apokalyptischen Literatur sowohl präsentische als auch futurische eschatologische Konzepte ihren Niederschlag finden, dann ist der Blick auf prophetische Konzepte im literarischen Umfeld nicht fern. ${ }^{53}$ Beide Konzepte stehen zwischen

47 Ibid., 5.

48 Ibid., 5.

49 Ibid., 6.

50 Ibid., 8. Mit dieser Definition unterscheiden sich die Autoren von einem Diskurs, der vor allem die Eschatologie der Apokalypse als warnende Zukunftsweisung verstand. Jörg Frey führt hierzu aus: „Die Eschatologie, und hier vor allem die futurische Komponente der eschatologischen Erwartung, hat dieses Buch auf der einen Seite zur Quelle der Erlösungshoffnung und Jenseitssehnsucht werden lassen, auf der anderen Seite konnte es dadurch auch für die Wahrnehmung vieler als eine Ankündigung von zu erwartenden Katastrophen bzw. des kommenden Weltuntergangs erscheinen - mit der Folge, dass der Begriff , apokalyptisch' im allgemeinen Sprachgebrauch inzwischen ausschließlich mit dieser negativen Konnotation gebraucht wird.” Frey, „Johannesapokalypse”, 478.

51 Aune, Prophecy, 323 geht nicht davon aus, dass die neutestamentlichen Gerichtsankündigungen von weitreichender hermeneutischer Bedeutung sein könnten, da „the announcement of judgment, the most common form of prophetic speech in the OT, is exceedingly rare in early Christianity. In early Christian prophetic traditions the threat of judgment is usually conditional, and usually occurs in conjunction with conditional promises of salvation".

52 Frey, „Johannesapokalypse”, 487-93.

53 Zu Mk 13 hält Eve-Marie Becker fest, dass „auch im Umkreis des frühen Christentums Geschichte, Prophetie und Eschatologie in die apokalyptischen Sprach- und Denkformen, die maßgeblich durch das Danielbuch vorgegeben waren, eingekleidet wurden". 
der Bewältigung der Vergangenheit und der Erwartung der zukünftigen Ereignisse. Tatsächlich finden sich Überlagerungen bzw. redaktionelle Überarbeitungen antiker Texte, die sowohl prophetische als auch apokalyptische Traditionen abbilden und damit keine widersprüchlichen Aussagen machen. ${ }^{54}$ Es wäre daher nicht sinnvoll, eine Trennung beider Deutungen der Vergangenheit, Gegenwart und Zukunft vorzunehmen. Eine Unterscheidung lässt sich also nicht zwischen dem prophetischen bzw. apokalyptischen Texten aufgrund ihres Anspruchs machen. Der Unterschied liegt vielmehr darin, dass die Apokalypse eine Visionszyklus ist, der „trotz des Wissens um das perfectum des Heilsgeschehens das futurum der Heilsvollendung klar akzentuiert" ${ }^{55}$ Die innergeschichtliche Naherwartung und die Enthüllung der gegenwärtigen Wirklichkeit dienen folglich der Antizipation einer Heilsvollendung. Wie sich bei den prophetischen Texten des Neuen Testaments gezeigt hat, legen diese den Fokus viel deutlicher auf die Sinndeutung gegenwärtiger Situationen. Die Gegenwart ist in diesem Sinn nicht enthülltes, sondern interpretiertes Objekt.

In Hinblick auf Apg 13.6-12 kann festgehalten werden, dass die Bewältigung der Vergangenheit und die Erwartung der zukünftigen Ereignisse in den Auswirkungen auf Barjesus sowie in der Reaktion des Prokonsuls ausgedrückt werden. Rainer Reuter vergleicht Apg 13 mit Apg 8 und bestätigt diese Schlussfolgerung:

Compared to Acts 8, the resistance of the magician is clearly increased, and because of that also the response of the Christian missionaries. It may well be that this is a literary technique. It enables the author to show the reader that fighting against contemporary magic is not at all a peripheral or harmless matter but a fight against evil, which is connected to the fading rule of Satan. At the same time, the author offers the clear message that evil and magic are going to be defeated by Christ and his power. ${ }^{56}$

Reuters Hinweise sind eindeutig: Der Kampf gegen die zeitgenössische Magie ist keine periphere oder harmlose Angelegenheit, sondern ein Kampf gegen das Böse und das Böse und die Magie werden durch Christus und seine Macht besiegt. Das perfectum des Heilsgeschehens und das futurum der Heilsvollendung liegen folglich auch in Apg 13.6-12 nah beieinander.

E.-M. Becker, „Markus 13 re-visited”, Apokalyptik als Herausforderung neutestamentlicher Theologie (Hg. M. Becker und M. Öhler; Tübingen: Mohr Siebeck, 2006) 108.

54 Siehe ibid., 124. Becker versteht Mk 13 als apokalyptische Redaktion prophetischer Tradition. 55 Frey, „Johannesapokalypse”, 546.

56 R. G. H. Reuter, „Animosity against Jewish and Pagan Magic in the Acts of the Apostles”, Animosity, the Bible, and Us: Some European, North American, and South African Perspectives (Hg. J. T. Fitzgerald, F. Van Rensburg, H. F. Van Rooy; Atlanta, GA: Society of Biblical Literature, 2009) 131. 


\subsection{Magie}

Die Definition von Magie ist nicht zuletzt von einer kulturwissenschaftlichen Unsicherheit hinsichtlich des „Gegenstandsbereiches Magie” geprägt. ${ }^{57}$ $\mathrm{Zu}$ viele Definitionen und Uneinigkeiten des Magiediskurses prägen die geisteswissenschaftliche Auseinandersetzung mit dem Begriff, sodass es Otto in seiner Monographie zur Magie sinnvoll erschien, „die fundamentalen heuristischen Probleme des Begriffs im Wissenschaftsdiskurs” zu betrachten. ${ }^{58}$ Was das Neue Testament betrifft, so spiegeln sich dies Schwierigkeiten bereits in den wenigen Bezügen zur Magie bzw. zu Magiern wieder. Otto hält fest, dass die neutestamentliche Rezeption des Magiebegriffs zwar ambivalent ist, weil in Mt 2.1 die Begriffe positiv verwendet werden, während Apg 8.9 sowie 13.6 sie negativ gebrauchen, aber dadurch analog zu seiner bisherigen Verwendung im griechischen Raum erfolgt. Er fasst zusammen, dass das Wirken Jesu gleichwohl von mageía abgesetzt wird und primär als Zeichen ( $\sigma \eta \mu \varepsilon i ̂ v)$, häufig auch als Kraft ( $\delta v \dot{v} \alpha \mu \iota \varsigma)$, seltener als Wunder $(\theta \alpha \hat{\mu} \mu \alpha)$ bezeichnet wird. ${ }^{59}$

Otto geht davon aus, dass die Ambivalenz, die sich hier im „dualistischen Sprachspiel” zwischen Magie und Wunder ausdrückt, terminologisch die frühchristlichen Abgrenzungsstrategien gegenüber dem konkurrierenden religiösen Umfeld bezeugt. ${ }^{60}$ Dies wird nicht nur dadurch begünstigt, dass im klassischen griechischen Sprachgebrauch Magie negativ konnotiert wäre, sondern dadurch verstärkt, dass keine terminologisch kohärenten Konzepte für die eindeutige Unterscheidung von Magie-, Wunder- und Prophetieterminologie vorlag. ${ }^{61}$ Dieser Sachverhalt wird nicht zuletzt an Apg 13.6-7 deutlich, denn hier wird der Jude Barjesus sowohl als Magier als auch als falscher Prophet bezeichnet. Es geht in dem Konflikt aber nicht um seine Magie, sondern darum, dass er den Statthalter von Paphos daran hindern will, das Wort Gottes zu hören. ${ }^{62}$ Apg 13.6f macht nicht nur auf die terminologische Nähe der Begriffe Magie und Prophetie aufmerksam, sondern auch auf die polemische Spannung zwischen religiösen Autoritäten. Barjesus steht für die Gefahr, dass der Statthalter zum einen die falsche Lehre hört und zum anderen der Chance beraubt wird, den Lehren der richtigen Propheten (Barnabas und Saulus) zu folgen. Dieses Bild könnte dem antiken Leser bekannt vorkommen. Naomi Janowitz fasst zusammen,

57 B.-C. Otto, Magie: Rezeptions- und diskursgeschichtliche Analysen von der Antike bis zur Neuzeit (Religionsgeschichtliche Versuche und Vorarbeiten 57; Berlin: De Gruyter, 2011) 1-2. 58 Ibid., 39. So auch N. Janowitz, Magic in the Roman World: Pagans, Jews and Christians

(Religion in the First Christian Centuries; London: Routledge, 2001) 5: „The term 'magic', in short, was too closely intertwined with polemics in the ancient world to easily, or even with a great deal of contortion, fall into a neat scholarly category."

59 Otto, Magie, 286.

60 Ibid., 286.

61 Zur rabbinischen Klassifikation der Magie siehe auch Janowitz, Magic, 20-6.

62 Otto, Magie, 284. 
dass im Alkibiades die magi für die Anbetung verantwortlich waren (1.122A), Dio Chrysostomos die magi als jene beschriebt, die in der Anbetung spezielle Kenntnisse hatten (Orationes 36.4), Apuleius in seiner Apologie Magier mit Priestern vergleicht (Apologia 25-6) ${ }^{63}$ und Philon ihnen unterstellt, die Wahrheit erkennen zu wollen. ${ }^{64}$ Mit Janowitz wird zum Magiebegriff ersichtlich, dass „[the] use by Jews, Christians and Greco-Roman writers was highly rhetorical, and pointed to the complex social textures of inter- and intragroup hostilities". ${ }^{65}$ Ein weiterer Aspekt der Magie, der mit der Vorstellung von Prophetie verglichen werden kann ist die Übermittlung von Wissen oder geheimen Kräften. Georg Luck beschreibt Magie als „eine Technik, die auf dem Glauben an geheime Kräfte im Menschen und im Weltall beruht, Kräfte, die unter besonderen Voraussetzungen vom Menschen geweckt und gelenkt werden können". ${ }^{66}$ Luck zeigt zudem auf, dass diese Technik eine Wechselwirkung zwischen dem Menschen und dem Weltall voraussetzt und dass der Magier in der Lage sei, die kosmischen Kräfte wie ein Medium zu vermitteln und weiter zu leiten. ${ }^{67}$ Wenn es um das Verhältnis zwischen Magie und Religion geht, hält Luck fest, dass Magie und Religion verwandte Phänomene sind und nicht klar voneinander $\mathrm{zu}$ trennen sind. ${ }^{68}$ Was sie allerdings trennt, und das ist bezüglich der Rolle prophetischer Rede interessant, ist die Beobachtung, dass Magie zwar auf einer Weltanschauung fusst, diese aber nicht ohne weiteres greifbar ist. ${ }^{69}$ Luck unterstellt Magiern also einen gewissen Zweckoptimismus und keine religiöse Interpretationsfähigkeit. Zweckoptimistisch ist ein Magier deshalb, weil er, nach Luck, selbstbewusst in die Zukunft schauen kann, da er damit rechnet, auch bei einem religiösen oder politischen Machtwechsel die kosmologischen Kräfte bündeln zu können. Der (alttestamentliche) Prophet ist im Gegensatz dazu unfreiwillig an die Botschaft des einen Gottes gebunden..$^{70}$ Diese zuweilen plakative Gegenüberstellung von Personen, die ihren Umgang mit den göttlichen Kräften finden und mit ihrer Umwelt teilen, weist unlängst darauf hin, dass weltanschauliche Differenzen bereits mit der Klassifizierung beginnen.

Teil der Rhetorik in Apg 13.6-12 ist es, dass Paulus sich scharf gegen den Magier abgrenzt. Worum geht es bei dieser Abgrenzung? Die Terminologie in

63 ... Nam si, quod ego apud plurimos lego, Persarum lingua magus est qui nostra sacerdos, quod tandem est crimen, sacerdotem esse et rite nosse atque scire atque callere leges cerimoniarum, fas sacrorum, ius religionum ....

64 Janowitz, Magic, 10.

65 Ibid., 6.

66 G. Luck, Magie und andere Geheimlehren in der Antike (Kröners Taschenausgabe; Stuttgart: Alfred Kröner, 1990) 1.

67 Ibid., 1.

68 Ibid., 6, 8.b

69 Ibid., 6.

70 Ibid., 290. 
Magie-, Wunder- und Prophetieberichten liegt sehr nah beieinander und wird nicht unbedingt gegeneinander ausgespielt. Auffällig ist in Apg 13.6-12, dass Unterschiede von Paulus hingegen bezüglich der Handlungsorientierung des Magiers gemacht werden. Er ist voller List und Tücke, er wird von Paulus als Sohn des Teufels bezeichnet, der ein Feind aller Gerechtigkeit ist. Den Magier als Pseudopropheten $\mathrm{zu}$ charakterisieren unterstreicht die lukanische Annahme, dass Paulus an dieser Stelle vor einem konkreten Problem steht. Im Vergleich mit weiteren Belegen des Neuen Testaments, bei denen über die sog. falschen Propheten berichtet wird, fällt der Blick auf Mt 7.15-23, denn hier wird auch vor den falschen Propheten gewarnt. Bernd Kollmann historisiert die matthäische Skepsis gegenüber Wunderheilern: „Mt steht grundsätzlich wundercharismatischen Strömungen seiner Zeit äußerst reserviert gegenüber. Maßgeblicher Grund dafür dürfte ein pneumatischer Enthusiasmus einzelner Wundercharismatiker im Umfeld des Mt-Ev gewesen sein, der sich in ,Pseudoprophetie' manifestierte (Mt 7,15-23; 24,23-28). ${ }^{71}$ Kollmann sieht die Kritik an den Pseudopropheten in engem Zusammenhang mit der Endgerichtsszene in Mt 7.21-3, die auch die Existenz eines Charismatikertums kritisiert, das Prophetie und Wundertaten miteinander verbindet. ${ }^{72}$ Auch hier besteht die Möglichkeit, dass innerhalb der christlichen Gemeinschaft einzelne kritisiert werden, die mit prophetischer Rede auch noch Dämonen austreiben, Wunder vollbringen und Kranke heilen (vergleichbar mit 1 Kor 12.9-10, 28-30). ${ }^{73}$ Wenn dem so ist, dass Mt das „Tun des väterlichen Willens” über die „prophetisch-ekstatischen Äußerungen" stellt, ${ }^{74}$ die auch mit deiktischen Handlungen verknüpft sind, dann spiegelt sich hier ein Diskurs wider, der zwischen verschiedenen Formen der Nachfolge unterscheidet. Mk 8.12, Jesu Verweigerung eines Zeichens, fügt sich in diese frühchristliche Debatte ebenso ein. Hier kann dem Text entnommen werden, dass Jesus „seine Wunder nicht als demonstrative Beglaubigungszeichen im Dienste von Verkündigung oder Lehre verstanden wissen will, sondern ihnen dem gegenüber in unmittelbarem Zusammenhang mit der Durchsetzung der Gottesherrschaft eigenständige Bedeutung zukommt". ${ }^{75}$ Es lässt sich folglich festhalten, dass bei der Diskussion über Prophetie und (magische) Zeichenhandlungen nicht die Interpretation des göttlichen Willens entscheidend ist, sondern die unterschiedliche Demonstration der göttlichen Vollmacht. In diesem Sinn zeigt sich bei der

71 B. Kollmann, Jesus und die Christen als Wundertäter: Studien zu Magie, Medizin und Schamanismus in Antike und Christentum (FRLANT; Göttingen: Vandenhoeck \& Ruprecht, 1996) 320.

72 Ibid., 330.

73 Ibid., 334 .

74 Ibid., 334.

75 Ibid., 285. 
neutestamentlichen Kritik an Prophetie und Magie ein Themenspektrum, das die Demonstration göttlicher Macht fokussiert.

Am Beispiel der Rhetorik in Apg 13.6-7 kommentiert Bernhard Heininger, dass die negative Konnotation der Magie durch den Textbefund nicht unbedingt bestätigt wird:

Denn mit Ausnahme des Umstands, dass Barjesus sich Paulus und Barnabas entgegenstellt, um - wie es im Munde des Paulus und in gut biblischer Diktion heißt - die geraden Wege des Herrn krumm zu machen (Apg 13,10), kommt es von Seiten des Barjesus zu keiner feindseligen, geschweige denn magischen Aktion, sodass die paulinischen Anwürfe („voll jeglicher List und Schlechtigkeit, Sohn des Teufels, Feind jedweder Gerechtigkeit”) dem neutralen Beobachter reichlich überzogen vorkommen. Wenn jemand in Apg 13,6-12 Schaden stiftet, dann ist das Paulus! Dem Textbefund entspricht das allerdings nicht unbedingt. ${ }^{76}$

Die Fluchrede des Apostels als „Schaden” zu bezeichnen ist nach den eben erwogenen Zusammenhängen fraglich. Vielmehr konnte festgestellt werden, dass die apokalyptische Rede des Apostels an Barjesus ein Model darstellt, mit dem Lukas eine Wirklichkeitserfahrung in einem bestimmten Offenbarungsgeschehen verorten kann. Lukas lässt den Leser folglich an der Antizipation einer Heilsvollendung teilhaben, so dass die Erblindung des Barjesus und die Konversion des Prokonsuls das perfectum des Heilsgeschehens und das futurum der Heilsvollendung gleichermassen ausdrücken. Es bestätigt sich: Der jüdische Pseudoprophet und Magier wird nicht aufgrund seiner religiösen Zugehörigkeit bedroht, sondern, weil er nicht aufhört, die geraden Wege des Herrn zu verdrehen und den Prokonsul daran hindern will, die Worte der christusgläubigen Apostel zu hören. Die unterschiedliche Demonstration der göttlichen Vollmacht, die auf die Heilsvollendung hinweist, ist das Differenzkriterium zwischen Paulus und Barjesus. In diesem Sinn wird in Apg 13.6-12 deutlich, dass Barjesus in der Antizipation einer Heilsvollendung nicht die richtigen Mittel wählt, um göttliche Vollmacht zu demonstrieren.

\section{Zusammenfassung}

Die Kontextualisierung von Apg 13.6-12 in die lukanische Geschichtstheologie als auch im neutestamentlichen Diskurs von Apokalyptik und Magie hat gezeigt, dass sich die Kategorien nicht ausschliessen - sondern sich gegenseitig ergänzen. Angefangen bei der Beobachtung, dass in Apg 13.6-12 historisch plausible Beziehungen und Entwicklungen zwischen Personen zu Gunsten der

76 B. Heininger, „Im Dunstkreis der Magie: Paulus als Wundertäter nach der Apostelgeschichte”, Biographie und Persönlichkeit des Paulus (Hg. O. Wischmeyer, E.-M. Becker, P. Pilhofer; Tübingen: Mohr Siebeck, 2005) 276. 
frühchristlichen Gemeinschaftsbildung beschrieben werden, wurde darauf hingewiesen, dass Lukas „in seinem Werk keinen direkten, sondern einen primär theologisch vorstrukturierten Zugang zur Vergangenheit [vermittelt]". ${ }^{77}$ Bei der gezielten Verortung des Wortes Gottes in einer scheinbar vom römischen Reich dominierten Welt, konnte am Beispiel von Apg 13.6-12 gezeigt werden, dass über das Hinzuziehen von Magie und Apokalyptik der Akt der Offenbarung und die Übertragung der Offenbarung im gleichen Augenblick auftreten. Folgt man Hagenes Trias von Erinnerung, Kontinuität und Identität als Ausdruck der lukanischen Geschichtstheologie, dann gilt es in Apg 13.6-12 vor allem die funktionale Geschichtsschreibung zu beschreiben. Die funktionale Geschichtsschreibung zu beschreiben unterscheidet sich von dem Versuch, den Grad der Historizität zu bestimmen dadurch, dass keine konkreten Personen, sondern die Kombination aus informierenden und beschreibenden Sachverhalten erörtert wird. Die Kombination also, die im Erinnern „Zeitverlaufsvorstellungen” und die „Selbstvergewisserung der historisch Erzählenden” miteinander ins Verhältnis setzt. An Apg 13.6-12 konnte gezeigt werden, dass die Thematisierung von Apokalyptik und Magie in eine tragende funktionale Gedächtniskultur der Gemeinschaft in ihrer gegenwärtigen Befindlichkeit eingebettet ist, sodass dem Leser eine historisch plausible Rekonstruktion der urchristlichen Gemeinschaftsbildung geschildert wird. Chad Hartsock illustriert dies an dem Bild der Erblindung:

As Paul's first act as a commissioned missionary, it is significant that he begins with an episode regarding blindness, and this should be no shock to the reader - Jesus began his ministry by announcing that the gospel brings sight to the blind (Luke 4); Paul was blinded when he first encountered Christ on the road to Damascus (Acts 9); and the whole of Luke-Acts will end with a proclamation that the eyes of some of the Jews are blind and thus the message will be taken to the Gentiles (Acts 28). Here, Paul begins his career by encountering a magician who is misleading the proconsul, and Paul must expose the magician as spiritually bankrupt, a task which is accomplished by blinding Bar-Jesus. ${ }^{78}$

Der Bogen von Lk 4 über Apg 9.13 und Apg 28 und die thematische Stringenz ist auf dem Hintergrund der historisch plausiblen Rekonstruktion überzeugend dennoch steht in Apg 13.6-12 nicht die physische Beschreibung vom Sehen und Erblinden im Vordergrund, sondern die unterschiedliche Demonstration der göttlichen Vollmacht. Die Drohung des Apostels und die darauf folgende

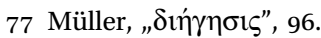

78 C. Hartsock, Sight and Blindness in Luke-Acts: The Use of Physical Features in Characterization (Biblical Interpretation series; Leiden: Brill, 2008) 199. Siehe auch für ein besseres Verstehen

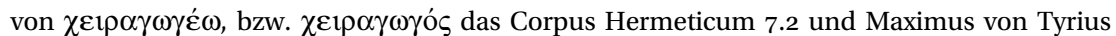
8.7h (T. J. Kraus, Ad fontes: Original Manuscripts and their Significance for Studying Early Christianity. Selected Essays (Texts and Editions for New Testament study; Leiden: Brill, 2007) 104). 
Erblindung des Pseudopropheten macht deutlich, dass nicht die Interpretation des göttlichen Willens entscheidend ist, sondern die unterschiedliche Demonstration der göttlichen Vollmacht. Schreiber fasst treffend zusammen: „Das Strafwunder dient also der Durchsetzung der Verkündigung." ${ }^{79}$ Dem ist aufgrund der hier dargestellten Bedeutung von Apokalyptik und Magie noch hinzuzufügen, dass das Bild folglich der funktionalen Gedächtniskultur einer Gemeinschaft dient. Magie und Apokalyptik stehen im Zentrum lukanischer Geschichtsschreibung zur Vergewisserung ihrer theologischen Einbettung in die Geschichte der Beziehung Gottes zu seinem erwählten Volk. Mit Hilfe von Magie und Apokalyptik wird trotz des Wissens um das perfectum des Heilsgeschehens das futurum der Heilsvollendung klar akzentuiert.

German abstract: Apg 13.6-12 wird im Zusammenhang mit der Historizitätsdebatte der Apostelgeschichte ein hoher Stellenwert zugesprochen, da hier die Anfänge der paulinischen Mission unter den Heiden vermutet werden und die Erzählung das paulinische Selbstverständnis als Apostel illustriert. Zudem werden gerade in diesem Abschnitt Anzeichen der literarischen Kreativität des Autors hinsichtlich des theologischen Gesamtkonzepts und der Thematisierung von Wundern, Magie und Apokalyptik deutlich, die der Darstellung der Anfänge der paulinischen Mission zu widersprechen scheinen. Dieser Aufsatz wird dem vermeintlichen Widerspruch in der Historizitätsdebatte des lukanischen Doppelwerks im Allgemeinen und Apg 13.6-12 im Besonderen nachgehen und zeigen, dass Magie und Apokalyptik durchaus im antiken Sinn von historischer Verifikation verstanden werden können.

79 Schreiber, Wundertäter, 30. 